\title{
Energy Efficiency Analysis of ICN Assisted 5G IoT System
}

\author{
Di Zhang, ${ }^{1,2}$ Zhenyu Zhou, ${ }^{1}$ Zhengyu Zhu, ${ }^{3}$ and Shahid Mumtaz ${ }^{4}$ \\ ${ }^{1}$ State Key Laboratory of Alternate Electrical Power System with Renewable Energy Sources, \\ School of Electrical and Electronic Engineering, North China Electric Power University, Beijing 102206, China \\ ${ }^{2}$ Information System Laboratory, Department of Electrical and Computer Engineering, Seoul National University, \\ Seoul 151-744, Republic of Korea \\ ${ }^{3}$ School of Information Engineering, Zhengzhou University, Zhengzhou 450001, China \\ ${ }^{4}$ Instituto de Telecomunicações, 1049-001 Aveiro, Portugal
}

Correspondence should be addressed to Zhenyu Zhou; zhenyu_zhou@ncepu.edu.cn

Received 26 August 2017; Accepted 5 December 2017; Published 26 December 2017

Academic Editor: Jun Huang

Copyright (C) 2017 Di Zhang et al. This is an open access article distributed under the Creative Commons Attribution License, which permits unrestricted use, distribution, and reproduction in any medium, provided the original work is properly cited.

\begin{abstract}
Other than separately investing the energy efficiency (EE) merits of information-centric networking's (ICN's) caching and sharing (CS) mechanism in wireless communications, here we comprehensively compare the EE performances of ICN's CS mechanism in different scenarios. A modified system model is first proposed while introducing the CS mechanism into the in-network router, base station (BS), and neighboring user sides. Afterwards, the system achievable sum rate as well as the power consumptions in wireless and wired sections is investigated. The EE performances of different scenarios are finally obtained by dividing the achievable sum rate by the consumed power. While comparing the three scenarios, numerical results demonstrate that the optimal place to cache the content is mainly determined by the distance and hub number of the core routers that passed.
\end{abstract}

\section{Introduction}

Fifth generation (5G) is calling for massive connected devices and a much faster transmission speed compared to prior long term evolution (LTE) [1]. In 5G, massive connected smart devices with uniquely identifiable computing and sensing ability [2] will play the vital role. Actually, 5G is aiming to connect everything from everywhere during every time with the big picture of Internet of Things (IoT). For instance, it is estimated that about 50 billion devices will be connected by the year of 2020 with various quality of service (QoS) requirements. In 5G IoT studies, highly functional smart device assisted communications attract increasing attentions. For instance, device (D2D) communication [3] was introduced to assist the wireless communications. With $\mathrm{D} 2 \mathrm{D}$, the users are enabled to communicate with each other directly. On the other hand, the intelligent vehicles [4] and other application scenarios become possible with the massive connected devices in $5 \mathrm{G}$.

In 5G IoT studies, energy efficiency (EE) [5] was arisen as another challenging issue besides the intensively studied spectrum efficiency (SE). This is mainly due to the fact that the even faster transmission speed and massive connected smart devices will consume more energy. Thus how to reduce the energy consumption and how to effectively use it are of great significance. In literature, a series of studies have been done on this issue by comprehensively optimize the transmission procedure $[5,6]$, turning off the unengaged devices of the transmission [7], and so on. On the other hand, the simultaneous wireless information and power transfer (SWIPT) [8], which is able to harvest the energy from the transmission procedure of neighboring user, attracts attentions from both academia and industry as well.

While prior work focused on the existing network architecture, recently, the information-centric networking (ICN) $[9,10]$ was proposed to redesign the network to cater to the driving force's shift of network evolution from the connection-centric to information-centric [11]. To this end, scholars claim on the Institute of Electrical and Electronics Engineers (IEEE) 5G summit in Silicon Valley that $5 \mathrm{G}$ cannot be simply achieved by the existing technologies heretofore. Redesigning the whole network architecture in both wireless and wired sections is inevitable. On the other hand, it is known that the user side only cares about the data no 
matter how and where it comes from. Meanwhile, with the massive connected smart portable devices, direct information delivery from the neighboring user side is possible. According to CISCO's report, the video traffic occupies 73\% in 2016 and will occupy $82 \%$ of the total information traffic in 2021. Thus how to effectively deliver the video and streaming data is vital in 5G IoT. To this end, it is believed that ICN will play a significant role in the forthcoming $5 \mathrm{G}$ IoT network architecture $[9,10]$.

The initial work on ICN, however, is mostly restricted to the upper layer and its applications for real time transmission and quality of experience (QoE) maintenance [12]. For instance, the proactive caching for wireless communications applications was investigated in the study of [13], in which the prototype system with hybrid text and contentcentric networking/named data networking (CCN/NDN) [11] protocols was proposed. It was demonstrated that highquality video delivery without interruption for up to 50 users simultaneously can be achieved by this method. An ICN based Ad Hoc network was proposed for disaster rescue in the study of [9]. The disaster information's publishing and retrieving can be accomplished within the connected areas even without network connection. A probabilistic caching was proposed to estimate the path and content caching ability to further improve the success hit in the study of [14]. Compared to the universal caching, this mechanism is able to reduce the cache-evictions by one order of magnitude. Additionally, ICN has been utilized in various regimes as well, for instance, the ICN/CCN/NDN based smart homes [15-17] and vehicular networks $[4,18]$.

Besides the prior work on upper layer and application research, ICN technology attracts intensive attentions in the study of EE. This is mainly because in that with caching and sharing (CS) mechanism, the distance and engaged components can be reduced, which yields less energy consumption while delivering the same amount of information [19]. In literature, the EE analysis with CS mechanism is to introduce the CS mechanism into the base station (BS) and neighboring user sides. For instance, caching at BS scenario was investigated in [19], in which the CS mechanism was introduced into the small cell with a mixed macro cell and small cell architecture. The system power consumption was reduced via this method. In [20], the content placement problem was discussed with heterogeneous network (HetNets) architecture. While analyzing the network performance and hit probability parameters, the optimal placement solutions were given. In the study of [21], by introducing the CS mechanism into neighboring user side, the optimal caching deployment was addressed according to transmit power and content popularity.

Although various studies have been done with ICN's CS mechanism on EE topic, it is found that the CS mechanism is separately discussed in prior work. A comprehensive performance comparison of obtaining the request contents from caches located in in-network router, BS, and neighboring user side is still in its fancy. That is, obtaining the request contents from where, under what specific condition, is still ambiguous. This inspires us to develop this treatise. To compare those three scenarios, it is assumed the request contents are distributed to the core router, BS, and neighboring users. Those distributions are defined as the core router, long distance, and short distance scenarios for the sake of convenient. The placement problem for obtaining the request content is finally addressed with the analysis and numerical results.

The contributions of this study are summarized as follows:

(i) A comprehensive redesigned system model is introduced for $5 \mathrm{G}$ IoT with the CS mechanism. That is, we introduce the caches into in-network router, BS, and the neighboring smart device sides with CS mechanism. With the smart portable devices, the user can obtain its request contents from neighboring user's caches. Additionally, it can also obtain the request contents from in-network router or BS caches.

(ii) The EE performance of core network, long distance, and short distance scenarios are investigated with achievable sum rate and consumed energy analysis. It is a versatile model that can be adopted by similar work as well.

(iii) Numerical results are used to answer the specific condition of where to obtain the request content problem. It is found that the short distance scenario has the best EE performance, followed by the long distance and core router scenarios. However, due to the limited battery of smart devices, in reality, long or core router scenarios are more reasonable choices.

The structure of this paper is organized as follows. Section 2 is the proposed system model. The CS mechanism is introduced to the user terminal, BS, and the core router. Achievable sum rate is investigated afterwards. The EE analysis is presented in Section 3. Section 4 is the numerical results. The paper is finally concluded by Section 5 .

\section{System Model}

The proposed system model as well as its sum rate analysis is addressed in detail in this section. Apart from prior studies, the CS mechanism is introduced to the neighboring user, core router, and the BS sides. The sum rate of the proposed system model is investigated afterwards.

2.1. Proposed System Model. Generally, communication system can be divided into the wireless section and wired section. Intuitionally, the wireless section can be taken as the end system that delivers the data request to (or download the request content from) the remote content server via the back-haul links connecting the core network [24, 25]. (Note that we focus on the content delivery from a remote content center to the user terminal; the other requests are not taken into consideration. The reason is that the majority of energy consumption is used for content data transmission, especially the video and similar streaming data [26].) In contrast, the wired section (i.e., core network, backbone network) consists of router, optical fiber link, remote content server, and so forth. 


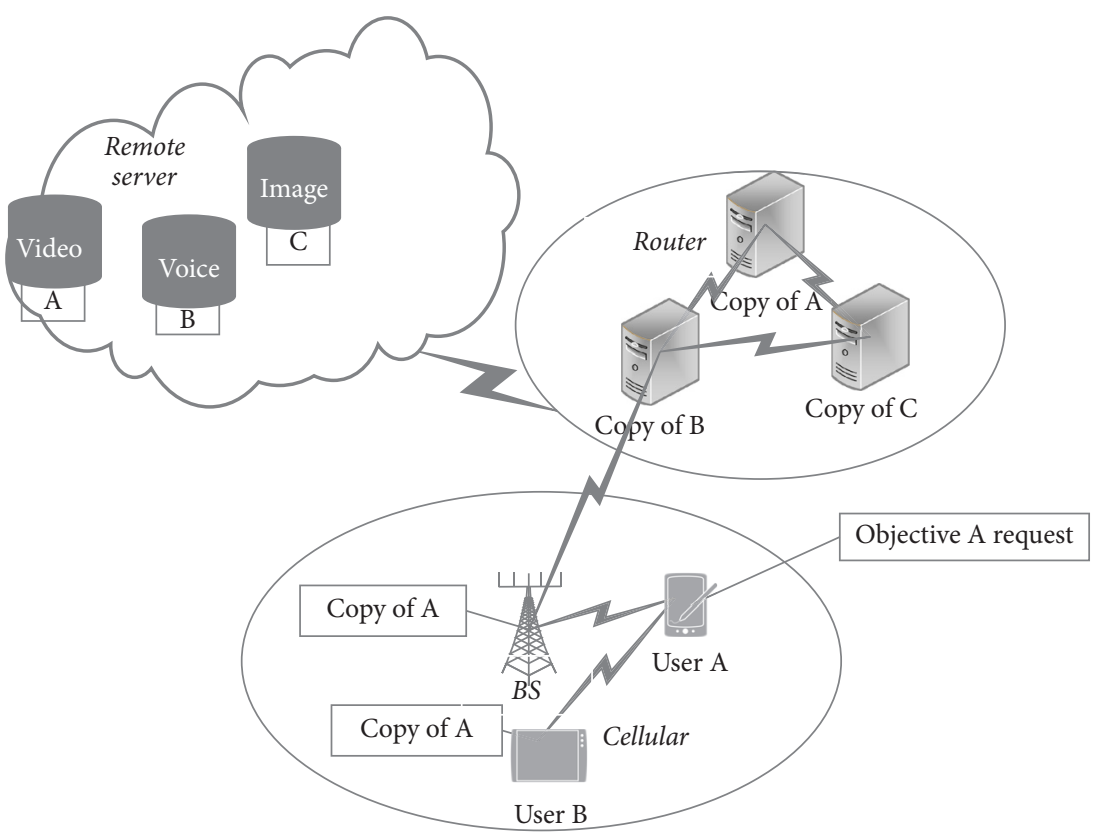

FIGURE 1: Description of the proposed system model.

As discussed before, with the incoming 5G IoT era, EE is becoming a challenge issue other than the well-studied SE topic. This is because the claiming even faster transmission rate will consume more energy compared with prior generations. The majority of studies of EE were to reduce the power consumption via effective transmission scheduling $[7,23,27]$. However, as discussed before, to accomplish 5G IoT's ambition while connecting massive devices with even faster transmission rate, redesigning the network architecture both from wired and wireless sections is needed. In light of this, an alternative system model with ICN technologies is introduced, which is given by the following parts.

In the proposed system here, massive MIMO antenna array is selected as the outdoor BS. It is assumed that one cell has 20 users with hundreds of massive MIMO antenna arrays, which is a widely used assumption of massive MIMO BS in 5G IoT [28]. The user's requested contents can be fulfilled by the contents storing in the caches of neighboring users with smart devices, BS, and the in-network core routers with CS mechanism. In contrast, the request contents can be directly retrieved from the remote content server (on condition that there is no requesting content cached in the cache). Detailed information of the optimized system is given by Figure 1. As shown, in the system, the CS concept is comprehensively introduced to the neighboring user, BS, and the in-network router sides. This is different from the prior literature of ICN that separately investigates the CS mechanism from "innetwork," BS, or neighboring user regimes.

Suppose there are two users within one cell area, user $A$ and user B, as shown in Figure 1. In addition, users A and $\mathrm{B}, \mathrm{BS}$, and the in-network routers in the wired core network are capable of caching and sharing the temporary hot contents (which are visited a lot). In this case, whenever user A has a content request, say "objective A request," it can be obtained from the caches named "Copy of A," in contrast to obtaining it from the remote content server as the conventional system model without the CS mechanism via back-haul links connected to the wireless and wired sections. Compared with obtaining it from the remote content server, the CS mechanism, once applied, can reduce the energy consumption via shorter distance and less components that are engaged in the transmission procedure, which yields better system EE performance. However, by what scale the EE performance will be enhanced is still ambiguous. Moreover, the decision should be clarified: say, under what constraint, distributing the contents and obtaining them from where within the constraint of this proposed system model should be set forth. This is the focused content distribution problem in this study, which will be answered by the following sections.

2.2. Sum Rate Analysis. Note that in this study, for the sake of compactness, we use the sum rate of one cell to denote the sum content request. Additionally, the user transmission speed is used to denote the content request of each user. That is, with a given volume of content request, by supposing that it can be satisfied within 1 second (generally, the request content volume will be around several hundreds of megabits; within the background of $5 \mathrm{G}$ by a claiming user experience rate up to $100 \mathrm{Mbit} / \mathrm{s} \sim 1 \mathrm{Gbits} / \mathrm{s}$ [29], it is possible to transmit such a volume of contents within 1 second; in addition, as the system EE performance is the focus, only the total rate and its consumed power are the targets of the analysis), the volume and transmission rate will be of the same quantity. Without loss of generality, equal transmission rate is adopted in user-to-user case, that without the massive MIMO effect, while gaining information from the other users' caches.

The EE analysis of long distance scenario mainly depends on the achievable sum rate and the needed energy for content delivery. It is first addressed here in this study. For the 
information delivery from BS to user side with massive MIMO, by assuming the channel matrix is $\mathbf{H}$, user number $N$, and antenna number $M$, the channel from transmit antenna to each user, such as user $k$, will be $\mathbf{h}_{k}$. In this case, channel matrix can be given as $\mathbf{H}=\left[\mathbf{h}_{1}, \mathbf{h}_{2}, \ldots, \mathbf{h}_{k}, \ldots, \mathbf{h}_{N}\right]^{T}$. Additionally, it is assumed that each vector $\mathbf{h}_{k}$ obeys the zero-mean complex-Gaussian entries with variance $1 / 2$ per dimension. As in prior studies, the zero-forced beam-forming (ZFBF) can perfectly cancel the cochannel interferences from other user $[7,30]$. It is adopted here with ZFBF matrix $\Omega$ :

$$
\begin{aligned}
\boldsymbol{\Omega} & =(\mathbf{H})^{H}=\mathbf{H}^{H}\left(\mathbf{H} \mathbf{H}^{H}\right)^{-1} \\
& \approx \frac{1}{M} \mathbf{H}^{H} \operatorname{diag}\left(\frac{1}{M} \mathbf{H} \mathbf{H}^{H}\right)^{-1} \\
& =\left[\frac{\mathbf{h}_{1}{ }^{H}}{\left\|\mathbf{h}_{1}\right\|^{2}}, \ldots, \frac{\mathbf{h}_{k}{ }^{H}}{\left\|\mathbf{h}_{k}\right\|^{2}}, \ldots, \frac{\mathbf{h}_{N}{ }^{H}}{\left\|\mathbf{h}_{N}\right\|^{2}}\right],
\end{aligned}
$$

where $\mathbf{A}^{H}, \mathbf{A}^{-1}$ are the Hermitian transpose and inverse transpose of a matrix $\mathbf{A}$, respectively. By denoting $\gamma$ as the normalization factor of signal of the $k$ th user, its expression can be given by $\gamma=\|\Omega\|_{F}^{2} / N$, where $\|\Omega\|_{F}^{2}$ yields the matrix Frobenius norm of $\Omega$. In this case, after the ZFBF, SINR expression of user $k$ will be $[7,30]$

$$
\operatorname{SINR}_{k}=\rho_{k} \frac{M-N}{N},
$$

where $\rho_{k}$ is the signal to noise ratio (SNR) of user $k$.

With SINR expression in hand, given constant carrier bandwidth, achievable transmit rate of each user $k$ can be given by following the Shannon theory $[7,30]$ :

$$
R_{k}=B \log _{2}\left(1+\operatorname{SINR}_{k}\right)=B \log _{2}\left(1+\rho_{k} \frac{M-N}{N}\right),
$$

where $B$ is the carrier bandwidth value. By further assuming $P_{k}, P_{n_{k}}$ the power of user $k$ and channel noise, respectively, the following equation holds:

$$
\rho_{k}=\frac{P_{k}}{P_{n_{k}}}
$$

In this case, by summarizing all the transmission rate within one cellular area, the achievable sum rate of one cellular area will be

$$
R_{\text {sum }}=\sum_{k=1}^{N} R_{k}=\sum_{k=1}^{N} B \log _{2}\left(1+\rho_{k} \frac{M-N}{N}\right) .
$$

Note that although the qualitative expression of $\rho_{k}$ has been given, the specific values of $P_{k}, P_{n_{k}}$ are still unknown. To settle down this, the following analysis will be employed.

\section{The EE Analysis}

EE analysis of different scenario is addressed here in this section. EE performances of the short and long distance scenarios are comprehensively analyzed due to the similar condition. In addition, it is assumed that the sum rate $\left(R_{\text {sum }}\right)$ is a constant value and equal to 20 users' request in the following analysis by following the typical massive MIMO BS configuration [31].

3.1. EE Analysis of Short and Long Distance Scenario. The quantitative analysis of $\rho_{k}$ within short and long distance scenarios is investigated beforehand. By following the free space path loss model, given a transmission distance $d$, received power at the receiver side can be given as [32]

$$
P_{r}=P_{t}\left[\frac{\sqrt{G_{l}} \lambda}{4 \pi d}\right]^{2}
$$

where $P_{t}$ is the power at massive MIMO BS antenna side. In addition, $\sqrt{G_{l}}$ yields the product of the transmitter and receiver antenna field radiation patterns in the light of sight (LOS) direction. (Here we assume that no non-light of sight (NLOS) path exists to simplify the analysis.) Moreover, $\lambda$ is the wavelength with expression

$$
\lambda=\frac{c}{f} .
$$

Here $c, f$ denote the transmission speed of light and carrier frequency, respectively. In line with prior study [33], giving carrier bandwidth, noise power is

$$
P_{n}=-174+10 \log _{10} B(\mathrm{dBm}) .
$$

According to the definition, SNR is the received target power divided by the noise power. This gives

$$
\rho_{k}=\frac{P_{k}}{P_{n_{k}}}=\frac{P_{t}\left[\sqrt{G_{l}} \lambda / 4 \pi d\right]^{2}}{P_{n}} .
$$

Thus while denoting constant sum rate value $R_{\text {sum }}$, we have the following equation:

$$
R_{\text {sum }}=\sum_{t=1}^{K} B \log \left[1+\frac{P_{t}\left[\sqrt{G_{l}} \lambda / 4 \pi d\right]^{2}(M-N)}{N P_{n}}\right] .
$$

As discussed, while the cached data are located in the massive MIMO BS and neighboring user sides, the requesting user can obtain its requested contents from caches of either neighboring user or BS side. Firstly, on condition that the requested content is obtained from the BS cache, which is the long distance scenario of this study, its power consumption can be estimated by

$$
P_{w}=P_{t, \text { total }}+P_{\mathrm{bs}}+P_{\mathrm{RF}}+P_{\text {circuit }} .
$$

Here $P_{t \text {,total }}$ denotes the power consumption of massive MIMO antenna array. According to prior study, it can be given by $P_{t \text {,total }}=\sum_{t=1}^{N} P_{t}$ [7]. Actually, it is the total power needed for all $N$ users' content delivery. Besides, $P_{\mathrm{bs}}, P_{\mathrm{RF}}$, $P_{\text {circuit }}$ are the power consumption of BS machine room (for instance, air conditioner) [34], radio frequency (RF) chain, and the circuit [22] to sustain such a transmission 
rate. It is worth noting that RF chain power consumption is around $100 \sim 200 \mathrm{~mW}$ [22], which is ignored here because of its negligible value compared to other component power consumption. Thus the power consumption of the long distance scenario can be given by

$$
P_{w} \approx P_{t, \text { total }}+P_{\mathrm{bs}}+P_{\text {circuit }} \text {. }
$$

In this case, given $d_{1}$, distance from request user to $\mathrm{BS}, \mathrm{EE}$ expression of the long distance scenario will be

$$
\eta_{\text {ee,long }} \approx \frac{R_{\text {sum }}}{P_{t, \text { total }}+P_{\mathrm{bs}}+P_{\text {circuit }}} .
$$

Here the approximate equation is due to the ignored RF chain power consumption.

Additionally, in the short distance scenario, the total request contents are assumed to be of the same value. This gives the same achievable sum rate value as in the long distance scenario while obtaining the request contents from the caches neighboring users. Moreover, within equal time period, the content request of each user results in equal transmission rate. Taking $d_{2}$ as the distance between request user and its neighboring user within one cellular area, since no BS or circuit is needed to engage the transmission in short distance scenario, by following the free space transmission procedure, the power consumption can be given as

$$
P_{s}=\frac{2^{\left(R_{\text {sum }} / B-1\right)} P_{n}\left(\sqrt{G_{l}} \lambda\right)^{2}}{\left(4 \pi d_{2}\right)^{2}} .
$$

By following the similar analysis of long distance scenario, EE performance of the short distance scenario will be

$$
\eta_{\text {ee }, \text { short }}=\frac{R_{\text {sum }}\left(4 \pi d_{2}\right)^{2}}{2^{\left(R_{\text {sum }} / B-1\right)} P_{n}\left(\sqrt{G_{l}} \lambda\right)^{2}} .
$$

Note that in short distance scenario, power threshold of user equipment (for instance, cell phone battery volume) typically is around $1 \sim 2 \mathrm{~W}$. It is impossible to sustain a transmission with consumed power larger than $P_{\mathrm{th}}$, even it has better EE performance. This is the constraint condition of short distance scenario, which will be used in the following analysis.

\subsection{EE Analysis of the Core Router Scenario. Other than} placing the content in the BS and neighboring user's caches in the wireless section, the content can be cached in the in-network core router side or, else, downloaded from the remote center as in previous architecture. EE analysis of core router scenario will be investigated with all engaged power consumptions taken into consideration in this subsection. It is noticed that in the with or without CS mechanism cases, their power consumptions can be given with a comprehensive expression; the only difference within the expression is the engaged hub and optical link numbers.

Generally, in wired section, participated equipment for information delivery consists of the asymmetric digital subscriber line, passive optical network, and fiber between the node [35]. Power consumption of the wired section in this case mainly comes from the network components (switcher, optical cross connect (OXC), etc.) and optical fiber (erbium doped fiber amplifier (EDFA), transmission, etc.). It can be given by the following expression:

$$
P_{c c}=\sum_{n=1}^{N_{n}}\left(P_{p}+P_{n, o}+P_{n, t 1}\right),
$$

where $N_{n}$ denotes the number of core network equipment items. Meanwhile, $P_{p}, P_{n, o}, P_{n, t 1}$ denote the power consumptions of one pair core network equipment, optical fiber, and the transmission, respectively. In addition, $P_{n}$ can be further divided as [36]

$$
\begin{aligned}
P_{n}= & P_{\text {trans }}+P_{\text {phy }}+P_{\text {mac }}+P_{\text {tp }}+P_{\mathrm{fi}}+P_{\text {mem }}+P_{\mathrm{lc}} \\
& +P_{\mathrm{ps}},
\end{aligned}
$$

where $P_{\text {trans }}, P_{\text {phy }}, P_{\text {mac }}, P_{\text {tp }}, P_{\text {fi }}, P_{\text {mem }}, P_{\text {lc }}, P_{\mathrm{ps}}$ yield the power consumptions of transceiver, physical layer (such as encoding/decoding, scrambling/descrambling, forward error correction (FEC)), Mac layer (such as mapping, framing), transport profile/forward error (TP/FE) (such as packet processing, classifying), fabric interference, line card, and the packet switch, respectively. On condition that only one line card is engaged in the transmission with all the others turned off to save energy, according to [36], one line card can sustain $40 \mathrm{GBits} / \mathrm{s}$ transmission speed, which is enough for this study due to the achievable sum rate value of wireless section. In addition, to be fair, the optimal wireless component selection and sleeping mechanism is adopted as well in the analysis while turning off the unneeded components. According to the estimation in [36], values of $P_{\text {trans }}, P_{\text {phy }}, P_{\mathrm{mac}}, P_{\mathrm{tp}}, P_{\mathrm{fi}}, P_{\mathrm{mem}}, P_{\mathrm{lc}}$, $P_{\mathrm{ps}}$ are $5.9 \mathrm{~W}, 3.4 \mathrm{~W}, 30.6 \mathrm{~W}, 183.6 \mathrm{~W}, 61.2 \mathrm{~W}, 13.6 \mathrm{~W}, 298.3 \mathrm{~W}$, $224.4 \mathrm{~W}$, respectively. Additionally, a simple EDFA structure is adopted with pumping, whereas its consumed power is $243 \mathrm{~W}$ taking into account that the pumping power is $97 \mathrm{~W}$ and the core pumped power $146 \mathrm{~W}$ [37]. This is the optical fiber power consumption by $P_{n, o}$ under ideal condition.

It is also noticed that the fan section (or cooling system) accounts of $33 \%$ of the total power consumption according to the estimation in $[38,39]$, which is ignored in prior studies $[36,37]$. In this work, to be closer to the reality, this amount power consumption is also taken into consideration, which gives the total power consumption in the core router section:

$$
P_{c c}=\sum_{n=1}^{N_{n}}\left(\frac{P_{p}}{67} 100+P_{n, o}+P_{n, t 1}\right) .
$$

While the core router delivers the request contents to the BS side, the wireless communications are invoked to handover the content to the user side from the BS. In this case, the power consumption while obtaining the request content in the core router scenario can be finally given by

$$
P_{c} \approx P_{c c}+P_{w} .
$$

Taking the hypothesis that the distance from router to router is $80 \mathrm{Km}$, the distance that one EDFA device can 
sustain is $80 \mathrm{Km}$ [40]. This distance is adopted in the analysis to simplify the analysis. Indeed, some other different distance values can be adopted, but this will not greatly affect the result since, in optical communication, the energy consumption mostly comes from the devices. Additionally, by normalizing the noise power $P_{n}$ to be 1 , taking into account that the transmission rate in wired section is equal to $R_{\text {sum }}$, the needed $P_{n, t 1}$ can be derived from the following equality:

$$
R_{\text {sum }}=B_{o} \log \left(1+P_{n, t 1}\right) .
$$

Here $B_{o}$ is the bandwidth of the optical fiber, typically taking the value of $10 \mathrm{GBit} / \mathrm{s}$. As we can see, the bandwidth is much larger than $R_{\text {sum }}$, which will result in a much lower power consumption of $P_{n, t 1}$ compared to the other equipment power consumption of the wired section, which is omitted here. This gives the EE performance of the core router scenario:

$$
\eta_{\mathrm{ee}, \mathrm{core}} \approx \frac{R_{\mathrm{sum}}}{P_{c}} .
$$

This is the EE performance expression of the core router scenario and without the CS mechanism by obtaining the request contents from remote center, as the only difference between the with and without CS mechanism scenarios in the core router side is the engaged router components and EDFA equipment. It is also worth noting that the optimal component selection is adopted in the analysis. Additionally, because of the small value of fiber power consumption, it is omitted here in this paper.

\section{Numerical Results}

The concerned caching location problem will be answered with the numerical results section. Here in the simulation, it is assumed that the requested content volume of each user is $200 \mathrm{MBit}$. On the other hand, taking the hypothesis that the request is satisfied within one second, a transmission rate $200 \mathrm{MBits} / \mathrm{s}$ is assumed. It is worth noting that the capacity and user experiment rate are different things. In $5 \mathrm{G}$, more than 1000-fold capacity increment of each cell is claimed compared to LTE, but generally, the user experiment rate is around $100 \mathrm{MBit} / \mathrm{s} \sim 1 \mathrm{GBit} / \mathrm{s}$ in its first phase [29].) In this case, achievable sum rate of the wireless section will be $4 \mathrm{GBits} / \mathrm{s}$ with 20 users (as discussed before, in typical massive MIMO BS area, there are hundred antennas serving around 20 users). Other related simulation parameters are given by Table 1 . The parameters and values are obtained by following the prior studies with $[7,34-37,41]$ as well as the 3GPP documents. Note that due to the inevitable dense cell deployment, in this simulation, $350 \mathrm{~m}$ cellular coverage area is used.

The prior optimal component selection studies in wireless communications (while obtaining the requested content from remote content center without the CS mechanism, such as the studies in $[22,23])$ are compared first with the core router scenario. Here the total core router hop is set to be 6 from BS to the remote content server ( 5 router hubs with 6 jumps from the BS to the server). For the sake of compactness, equal travel distance from the BS to the first router, router to router,
TABLE 1: Simulation parameters.

\begin{tabular}{lc}
\hline Parameter & Value \\
\hline Carrier frequency $f$ & $1900 \mathrm{MHz}$ \\
Carrier band $B$ & $20 \mathrm{MHz}$ \\
Antenna number $M$ & 100 \\
User number $N$ & 20 \\
Per user request & $20 \mathrm{MBit} / \mathrm{s}$ \\
Speed of light $c$ & $3 \times 10^{8} \mathrm{~m} / \mathrm{s}$ \\
$G_{l}$ & 1 \\
User power threshold $P_{\text {th }}$ & $2 \mathrm{~W}$ \\
BS range $d_{1}$ & $350 \mathrm{~m}$ \\
Machine room power $P_{\mathrm{bs}}$ & $400 \mathrm{~W}$ \\
Circuit power $P_{\text {circuit }}$ & $160.8 \mathrm{~W}$ \\
\hline
\end{tabular}

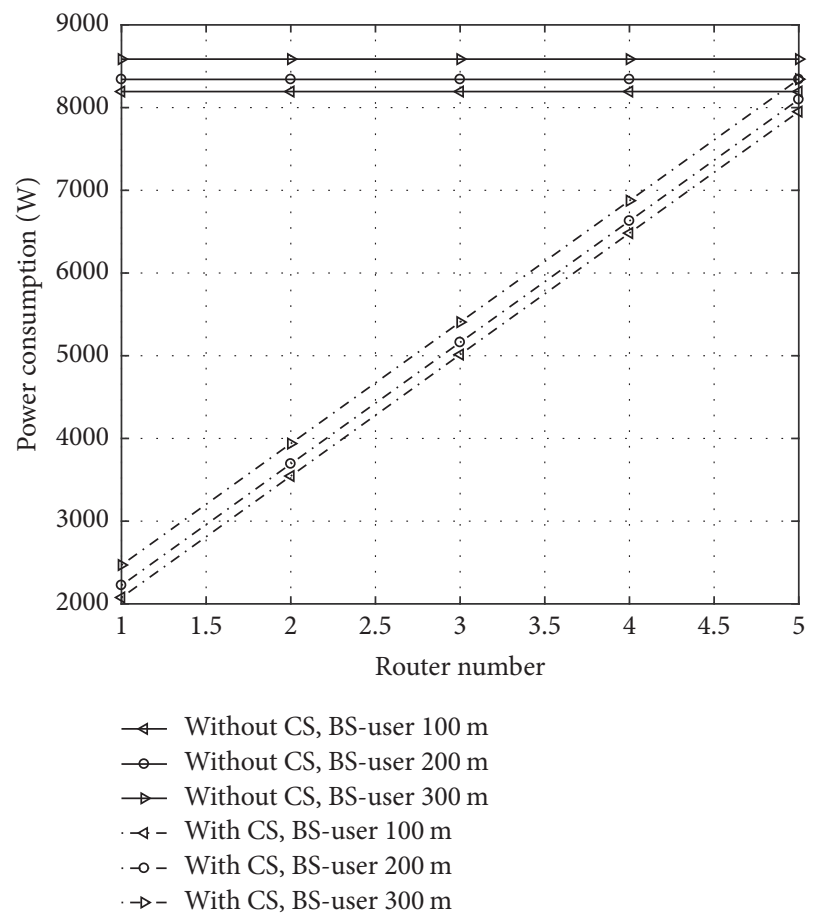

FIGURE 2: Power consumption comparison between the prior studies $[22,23]$ and the core router cache scenario. The calculation is according to (19) with simulation parameters given by Table 1 .

and the last router to remote content server is used as well. In core router scenario, it is assumed that the contents are cached by the first, second, third, fourth, and fifth router's caches. Moreover, to be fair, optimal engaged wireless and wired component numbers are used in both cases. Additionally, without loss of generality, it is assumed that the distance from BS to user side is $100,200,300 \mathrm{~m}$ in the core router scenario within the $350 \mathrm{~m}$ cellular coverage area.

The simulation results are given by Figures 2 and 3. As shown by the results here, core router scenario can reduce the power consumption and enhance the system EE performance compared with the prior studies in $[22,23]$. It is found that the less the router number content caches vary, the less the power consumption and the better the EE performance 


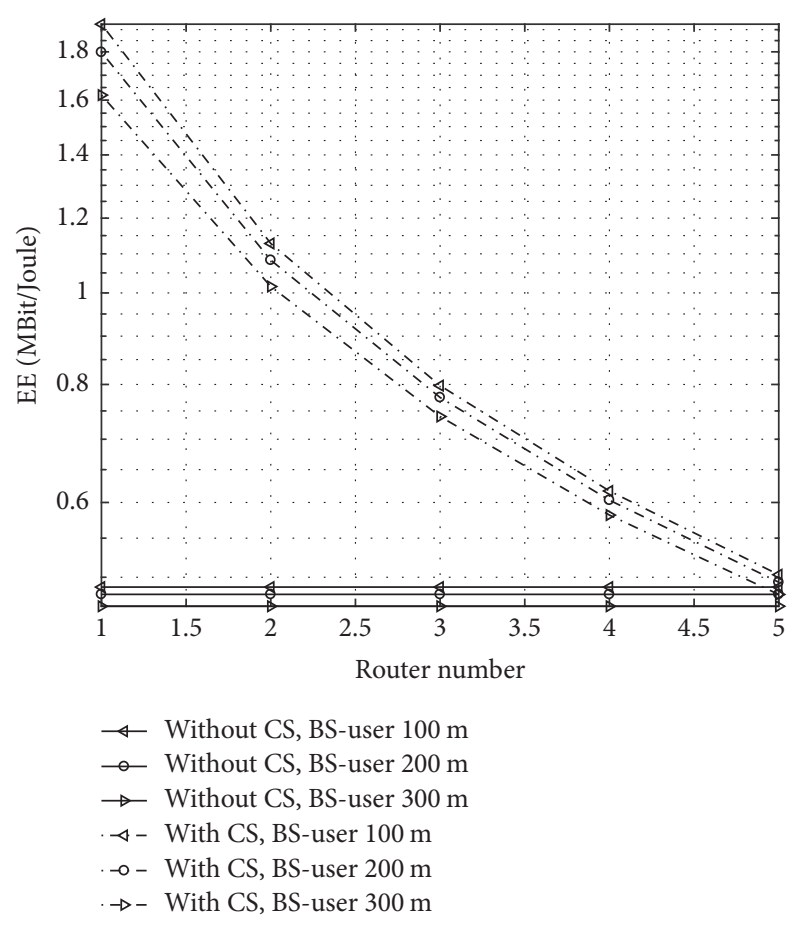

FIGURE 3: EE comparison between the prior studies [22, 23] and the core router cache scenario. The calculation is according to (21) with simulation parameters given by Table 1 .

are. On the contrary, with caching router approaching the remote center, its performance approaches the without cache scenario. This is because, with cached contents being near the remote content server, more engaged devices are needed with more consumed power, which narrows the difference between with and without the CS mechanism scenarios. On the other hand, increasing the distance from BS to user side yields more power consumption and worse system EE performance. This is mainly because the higher transmission loss in the environment requires more transmit power. The detailed consumed energy and EE performance with regard to the distance will be further discussed by the following long and short distance scenarios. It is also worth noting that due to the optical link as well as one EDFA between the last router and remote center, the power consumption and $\mathrm{EE}$ performance are different between obtaining the requested content from the last router's cache and obtaining it from the remote center.

The consumed power and EE performance of the long distance scenario are examined afterwards, where the results are shown by Figure 4. Furthermore, consumed power and EE performance of the short distance scenario are given by Figure 5. In the figures, " $m$ " of the $x$-axis means the distance (by meter) between the requesting user and contents (from neighboring user's caches or BS cache). It is clearly shown by Figures 4 and 5 that the short distance scenario can greatly save the energy consumption while showing a better EE performance. However, due to the threshold constraint of user equipment (typically $2 \mathrm{~W}$ ), the short distance scenario is only practical within a limited distance, around $0.2 \mathrm{~m}$
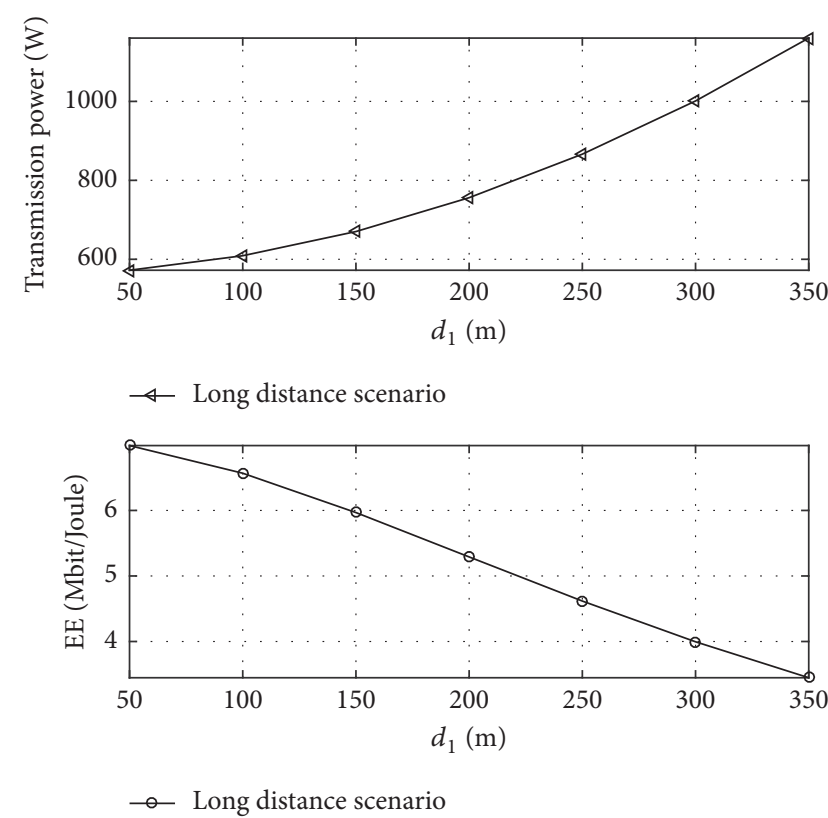

FIgure 4: Long distance scenario. The calculation is according to (12) and (13) with simulation parameters given by Table 1.

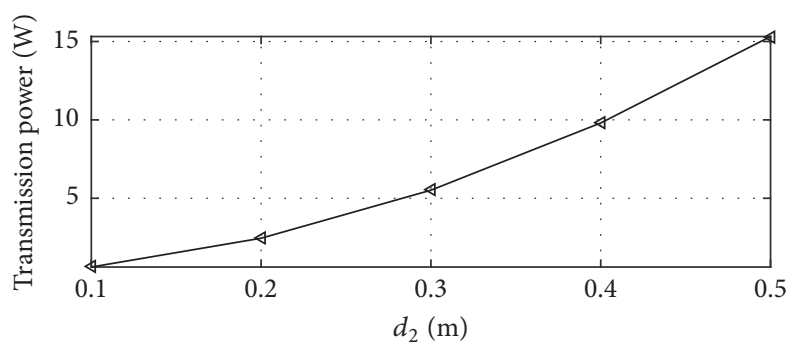

$\triangleleft$ Short distance scenario

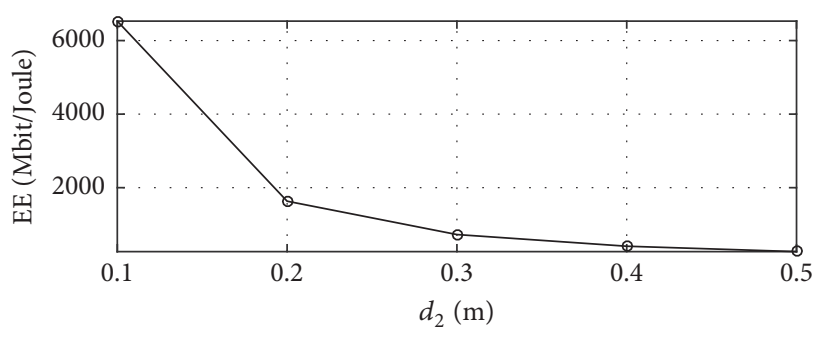

- $\rightarrow$ Short distance scenario

FIgURE 5: Short distance scenario. The calculation is according to (14) and (15) with simulation parameters given by Table 1.

as shown by this simulation. The short distance scenario then, while being applied in the outdoor environment, is almost impossible to adopt before the developed large volume battery or other solutions to overcome this bottleneck. In this regard, some groups are working on the large volume battery with graphene. Additionally, the existing technologies and studies with energy harvesting [42] and SWIPT [43] can partly alleviate the bottleneck. While summing up the simulation results, they demonstrate that normally the 
long distance scenario should be adopted in the outdoor environment. For some indoor applications or extreme short distance transmission, the short distance scenario can be a good choice. Besides, Figure 4 also shows that the consumed power is greatly increased while cellular range (from BS to the target user) is increasing. Sometimes this is even intolerable as a way of higher power consumption. For example, as shown by this figure, by range $350 \mathrm{~m}$, the energy consumption is about $1000 \mathrm{~W}$, which is almost equal to prior LTE BS power consumption with $1000 \mathrm{~m}$ coverage range. Here is another evidence of inevitable denser cellular deployment in 5G.

By comparing all the three scenarios, observation shows that the short distance scenario consumed the least power, following by the long distance scenario and core router cache scenarios. In this regard, the short distance scenario shows the best system EE performance, followed by the long distance scenario and core router scenario. All of the cache-enabled scenarios show better EE performance than prior studies. In this case, ICN's CS mechanism can reduce the power consumption and enhance the system $\mathrm{EE}$ performance. In addition, while adopting the CS mechanism, for distance less than $0.2 \mathrm{~m}$ (typically in the indoor environment), short distance scenario is a good choice with the best system EE performance. Generally in the outdoor environment, the long distance scenario can be a more feasible choice.

\section{Conclusion}

One comprehensive system model was introduced with CS mechanism in this paper. Based on the proposed system model, EE performance analysis was comprehensively investigated with in-network core router as well as short and long distance scenarios. Simulation results demonstrated that all the three scenarios showed better EE performance compared to the prior studies without the CS mechanism. While applying the CS mechanism in the outdoor environment, long distance scenario is a more reasonable choice. In the indoor and other scenarios with closer user-to-user distance, short distance scenario is better than the long distance scenario. Otherwise, the core router scenario is a feasible choice compared to that without CS mechanism.

\section{Conflicts of Interest}

The authors declare that there are no conflicts of interest regarding the publication of this paper.

\section{Acknowledgments}

This work was supported in part by the National Science Foundation of China (NSFC) under Grants nos. 61601180 and 61601181; the Fundamental Research Funds for the Central Universities under Grant no. 2016MS17; Natural Science Foundation of Beijing Municipality under Grant no. 4174104; Beijing Outstanding Young Talent under Grant no. 2016000020124G081; in part by the National Nature Science Foundation of China (NSFC) under Grants nos. 61571402, 61301150, 61571401, 61640005, and 61401401.

\section{References}

[1] D. Zhang, Z. Zhou, S. Mumtaz, J. Rodriguez, and T. Sato, "One Integrated Energy Efficiency Proposal for 5G IoT Communications," IEEE Internet of Things Journal, vol. 3, no. 6, pp. 1346$1354,2016$.

[2] J. Huang, Q. Duan, C.-C. Xing, and H. Wang, "Topology control for building a large-scale and energy-efficient internet of things," IEEE Wireless Communications Magazine, vol. 24, no. 1, pp. 67-73, 2017.

[3] J. Huang, Y. Sun, and Q. Chen, "GALLERY: a game-theoretic resource allocation scheme for multicell device-to-device communications underlaying cellular networks," IEEE Internet of Things Journal, vol. 2, no. 6, pp. 504-514, 2015.

[4] S. H. Bouk, S. H. Ahmed, M. A. Yaqub, D. Kim, and M. Gerla, "DPEL: Dynamic PIT entry lifetime in Vehicular named data networks," IEEE Communications Letters, vol. 20, no. 2, pp. 336339, 2016.

[5] K.-S. Koo and M. Govindarasu, "Energy-efficient opportunistic network coding algorithms for wireless networks," in Proceedings of the 24th International Conference on Computer Communications and Networks, ICCCN '15, USA, August 2015.

[6] H. Q. Ngo, E. G. Larsson, and T. L. Marzetta, "Energy and spectral efficiency of very large multiuser MIMO systems," IEEE Transactions on Communications, vol. 61, no. 4, pp. 1436-1449, 2013.

[7] D. Zhang, K. Yu, Z. Zhou, and T. Sato, "Energy efficiency scheme with cellular partition zooming for massive mimo systems," in Proceedings of the 12th IEEE International Symposium on Autonomous Decentralized Systems, ISADS '15, pp. 266-271, Taiwan, March 2015.

[8] J. Huang, C. Xing, and C. Wang, "Simultaneous wireless information and power transfer: technologies, applications, and research challenges," IEEE Communications Magazine, vol. 55, no. 11, pp. 26-32, 2017.

[9] Z. Wen, D. Zhang, K. Yu, and T. Sato, "Information centric networking for disaster information sharing services," IEICE Transactions on Fundamentals of Electronics, Communications and Computer Sciences, vol. E98A, no. 8, pp. 1610-1617, 2015.

[10] Z. Liu, M. Dong, B. Gu, C. Zhang, Y. Ji, and Y. Tanaka, "FastStart Video Delivery in Future Internet Architectures with Intra-domain Caching," Mobile Networks and Applications, vol. 22, no. 1, pp. 98-112, 2017.

[11] Z. Liu, M. Dong, B. Gu, C. Zhang, Y. Ji, and Y. Tanaka, "Interdomain popularity-aware video caching in future Internet architectures," in Proceedings of the 11th EAI International Conference on Heterogeneous Networking for Quality, Reliability, Security and Robustness, QSHINE 2015, pp. 404-409, Taiwan, August 2015.

[12] Z. Liu, Y. Ji, X. Jiang, and Y. Tanaka, "User-behavior driven video caching in content centric network," in Proceedings of the 3rd ACM International Conference on Information-Centric Networking, ACM-ICN 2016, pp. 197-198, Japan, September 2016.

[13] K. Kanai, T. Muto, J. Katto et al., "Proactive content caching for mobile video utilizing transportation systems and evaluation through field experiments," IEEE Journal on Selected Areas in Communications, vol. 34, no. 8, pp. 2102-2114, 2016.

[14] I. Psaras, W. K. Chai, and G. Pavlou, "Probabilistic in-network caching for information-centric networks," in Proceedings of the 2nd ACM SIGCOMM Information-Centric Networking Workshop, ICN '12, pp. 55-60, Finland, August 2012. 
[15] S. H. Bouk, S. H. Ahmed, D. Kim, and H. Song, "Named-datanetworking-based its for smart cities," IEEE Communications Magazine, vol. 55, no. 1, pp. 105-111, 2017.

[16] C. M. Park, R. A. Rehman, and B. Kim, "Packet Flooding Mitigation in CCN-Based Wireless Multimedia Sensor Networks for Smart Cities," IEEE Access, vol. 5, pp. 11054-11062, 2017.

[17] S. H. Ahmed and D. Kim, "Named data networking-based smart home," ICT Express, vol. 2, no. 3, pp. 130-134, 2016.

[18] S. H. Ahmed, M. A. Yaqub, S. H. Bouk, and D. Kim, "Towards content-centric traffic ticketing in VANETs: an application perspective," in Proceedings of the 7th International Conference on Ubiquitous and Future Networks (ICUFN '15), pp. 237-239, Sapporo, Japan, July 2015.

[19] S. Zhou, J. Gong, Z. Zhou, W. Chen, and Z. Niu, "GreenDelivery: Proactive content caching and push with energy-harvestingbased small cells," IEEE Communications Magazine, vol. 53, no. 4, pp. 142-149, 2015.

[20] J. Wen, K. Huang, S. Yang, and V. O. Li, "Cache-enabled heterogeneous cellular networks: optimal tier-level content placement," IEEE Transactions on Wireless Communications, vol. 16, no. 9, pp. 5939-5952, 2017.

[21] Y. Long, Y. Cai, D. Wu, and L. Qiao, "Content-related energy efficiency analysis in cache-enabled device-to-device network," in Proceedings of the 8th International Conference on Wireless Communications and Signal Processing, WCSP 2016, China, October 2016.

[22] D. Zhang, S. Mumtaz, Z. Zhou, and T. Sato, "Integrating Energy Efficiency mechanism with components selection for massive MIMO based C-RAN," in Proceedings of the IEEE International Conference on Communications Workshops, ICC '16, pp. 74-79, Malaysia, May 2016.

[23] A. Zappone, L. Sanguinetti, G. Bacci, E. Jorswieck, and M. Debbah, "A framework for energy-efficient design of 5G technologies," in Proceedings of the IEEE International Conference on Communications, ICC 2015, pp. 1845-1850, gbr, June 2015.

[24] J. Andrews and A. Gatherer, "Will densification be the death of 5G? , IEEE ComSoc CTN," Tech. Rep, May 2015.

[25] H. Lundqvist, "Death by starvation?" in Backhaul and 5G, IEEE ComSoc CTN, Death by starvation, Backhaul and 5G, 2015.

[26] J. Baliga, R. W. A. Ayre, K. Hinton, and R. S. Tucker, "Energy consumption in wired and wireless access networks," IEEE Communications Magazine, vol. 49, no. 6, pp. 70-77, 2011.

[27] H. Kim, C.-B. Chae, G. De Veciana, and R. W. Heath Jr., "Energy-efficient adaptive mimo systems leveraging dynamic spare capacity," in Proceedings of the CISS 2008, 42nd Annual Conference on Information Sciences and Systems, pp. 68-73, USA, March 2008.

[28] E. G. Larsson, O. Edfors, F. Tufvesson, and T. L. Marzetta, "Massive MIMO for next generation wireless systems," IEEE Communications Magazine, vol. 52, no. 2, pp. 186-195, 2014.

[29] "I.-. promote group, 5G network technology architecture white paper, May 2015”.

[30] M. Jung, T. Kim, K. Min, Y. Kim, J. Lee, and S. Choi, "Asymptotic distribution of system capacity in multiuser MIMO systems with large number of antennas," in Proceedings of the 2013 IEEE 77th Vehicular Technology Conference, VTC Spring 2013, Germany, June 2013.

[31] J. Vieira, S. Malkowsky, K. Nieman et al., "A flexible 100-antenna testbed for Massive MIMO," in Proceedings of the 2014 IEEE Globecom Workshops, GC Wkshps 2014, pp. 287-293, USA, December 2014.
[32] A. Goldsmith, Wireless Communications, Cambridge University Press, New York, NY, USA, 1st edition, 2005.

[33] Q. Gu, RF System Design of Transceivers for Wireless Communications, Springer-Verlag, New York, NY, USA, 2005.

[34] C. Lab, "Cloud radio network white paper," Tech. Rep, 2014.

[35] J. Baliga, R. Ayre, K. Hinton, W. V. Sorin, and R. S. Tucker, "Energy consumption in optical IP networks," Journal of Lightwave Technology, vol. 27, no. 13, pp. 2391-2403, 2009.

[36] S. Aleksi, "Analysis of power consumption in future highcapacity network nodes," Journal of Optical Communications and Networking, vol. 1, no. 3, Article ID 5207103, pp. 245-258, 2009.

[37] Y. Tsuchida, K. Maeda, K. Watanabe et al., "Cladding-pumped L-band multicore EDFA with reduced power consumption," in Proceedings of the Summer Topicals Meeting Series, SUM '14, pp. 148-149, Canada, July 2014.

[38] A. Greenberg, J. Hamilton, D. A. Maltz, and P. Patel, “The cost of a cloud: research problems in data center networks," Computer Communication Review, vol. 39, no. 1, pp. 68-73, 2009.

[39] A.-C. Orgerie, M. D. De Assuncao, and L. Lefevre, "A survey on techniques for improving the energy efficiency of large-scale distributed systems," ACM Computing Surveys, vol. 46, no. 4, article no. 47, 2014.

[40] X. Zhou and M. Birk, "Performance comparison of an 80-kmper-span EDFA system and a 160-km hut-skipped all-Raman system over standard single-mode fiber," Journal of Lightwave Technology, vol. 24, no. 3, pp. 1218-1225, 2006.

[41] E. E. Narimanov and P. Mitra, "The channel capacity of a fiber optics communication system: Perturbation theory," Journal of Lightwave Technology, vol. 20, no. 3, pp. 530-537, 2002.

[42] Z. Zhou, J. Gong, Y. He, and Y. Zhang, "Software defined machine-to-machine communication for smart energy management," IEEE Communications Magazine, vol. 55, no. 10, pp. 52-60, 2017.

[43] Z. Zhu, Z. Wang, Z. Chu, and D. Zhang, "Beamforming optimization via max-min SINR in MU-MISO SWIPT systems under bounded channel uncertainty," IEICE Transactions on Fundamentals of Electronics, Communications and Computer Sciences, vol. E99A, no. 12, pp. 2576-2580, 2016. 


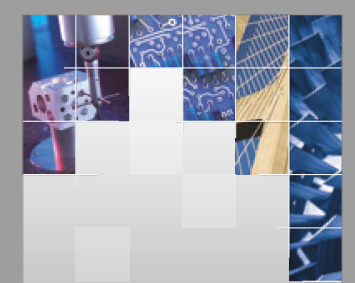

\section{Enfincering}
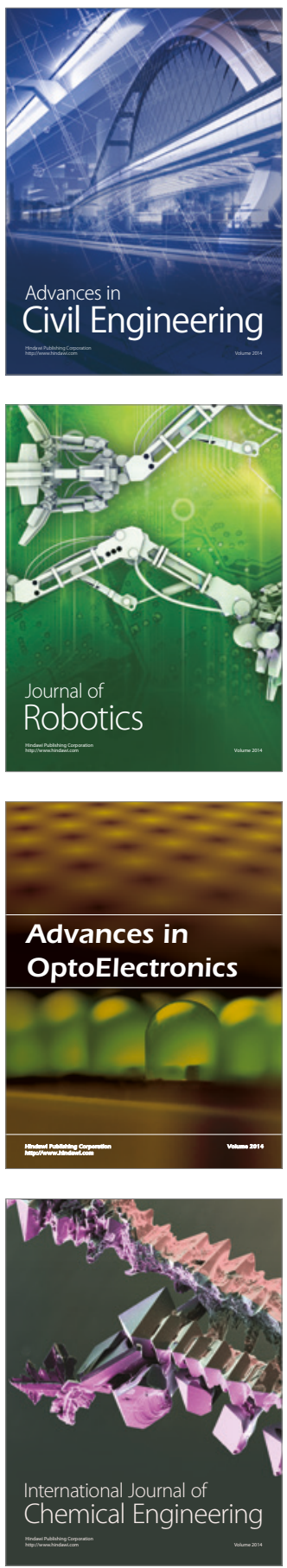

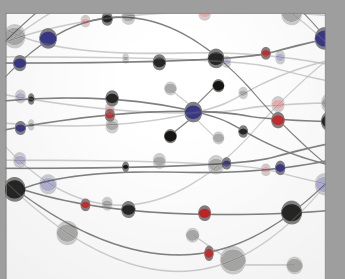

The Scientific World Journal

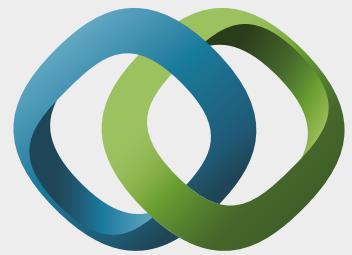

\section{Hindawi}

Submit your manuscripts at

https://www.hindawi.com
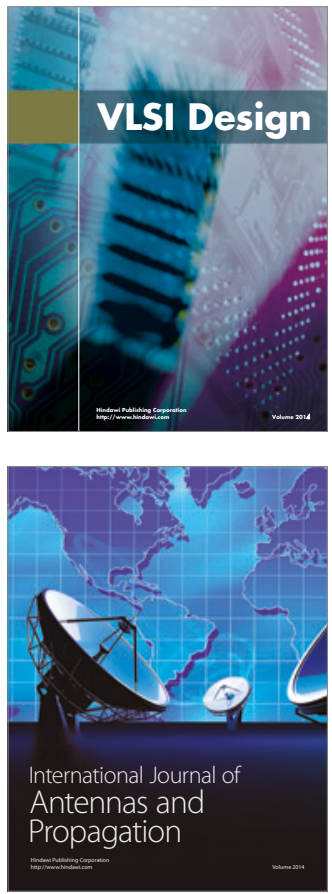

\section{Rotating}

Machinery
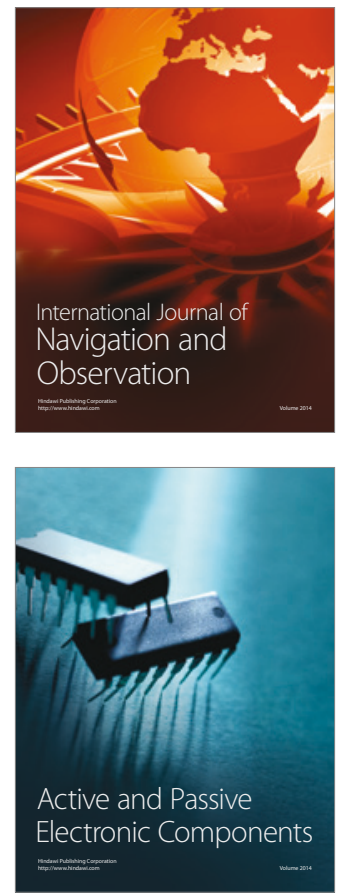
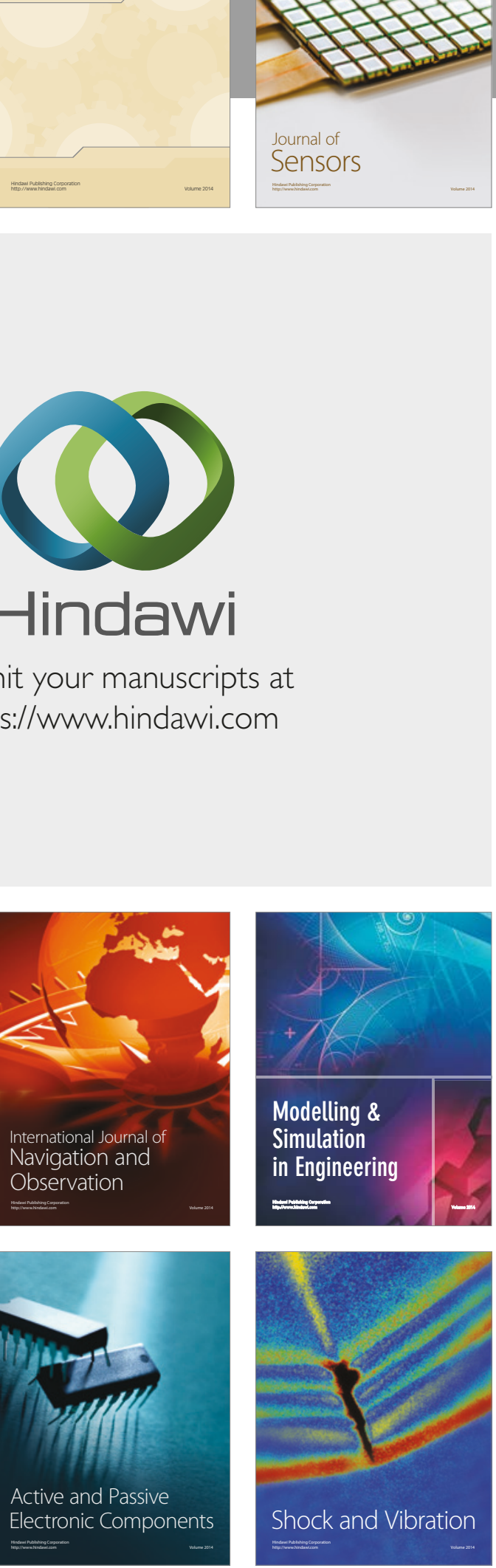
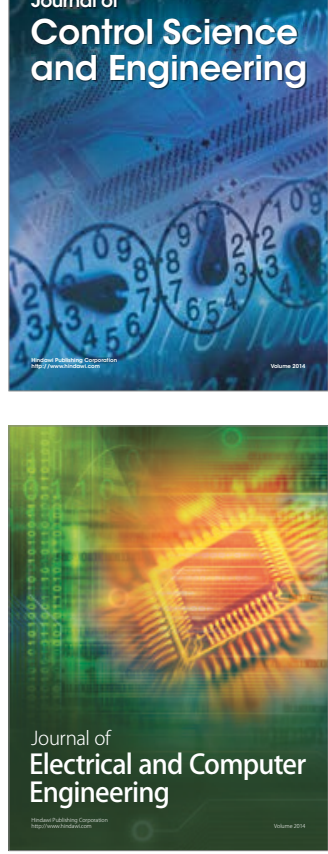

Distributed

Journal of

Control Science

and Engineering
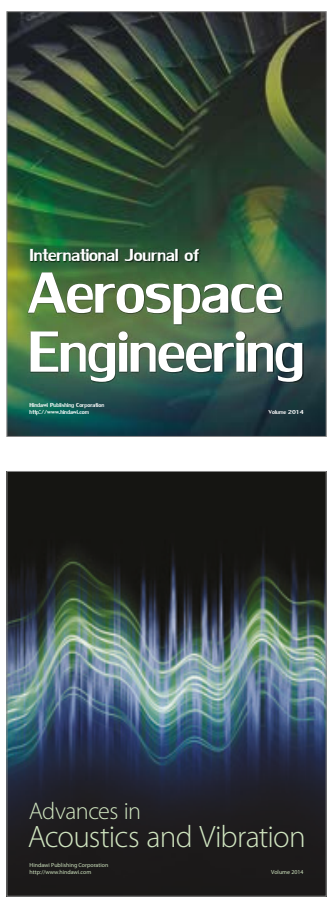

Sensor Networks 